\title{
The Association Between Hyperlipidemia and Periodontal Infection
}

\author{
Leila Golpasand Hagh ${ }^{1}$; Faramarz Zakavi ${ }^{2}$; Fatemeh Hajizadeh ${ }^{3,{ }^{*}}$; Morteza Saleki $^{4}$ \\ ${ }_{1}^{1}$ Department of Periodontics, School of Dentistry, Ahvaz Jundishapur University of Medical Sciences, Ahvaz, IR Iran \\ 2 Department of Operative Dentistry, School of Dentistry, Ahvaz Jundishapur University of Medical Sciences, Ahvaz, IR Iran \\ ${ }^{3}$ Department of Orthodontics, School of Dentistry, Ahvaz Jundishapur University of Medical Sciences, Ahvaz, IR Iran \\ ${ }^{4}$ Department of Pediatric Dentistry, School of Dentistry, Ahvaz Jundishapur University of Medical Sciences, Arvand Branch, Ahvaz, IR Iran \\ ${ }^{*}$ Corresponding Author: Fatemeh Hajizadeh, Department of Orthodontics, School of Dentistry, Ahvaz Jundishapur University of Medical Sciences, Golestan St., Ahvaz, IR Iran. Tel: \\ +98-9166317753, Fax:+98-6133730142, E-mail: hajizadeh_fatemeh@yahoo.com \\ Received: May 29, 2012; Revised: February 1, 2014; Accepted: October 25, 2014
}

\begin{abstract}
Background: Periodontitis is a local chronic inflammatory condition of the supporting structures of the teeth resulting from a dental plaque biofilm attached to teeth surfaces. Recent studies have indicated that this oral disease may have effects on systemic health.

Objectives: The aim of the present study was to evaluate the association between periodontitis and hyperlipidemia.

Patients and Methods: This case-control study was conducted in Iran during March 2011. In this case-control study, levels of serum lipids in 45 subjects with periodontitis were measured and compared with 45 age, gender and body mass index (BMI) matched controls. Data were analyzed using student $t$-test and chi-square test with $\mathrm{P}<0.05$ as the limit of significance.

Results: Mean values of total cholesterol $(\mathrm{CHL})$ (periodontitis group $=218.11 \pm 29.7$, control group $=162.31 \pm 48.24$ ) and triglycerides $(\mathrm{TG}$ ) (periodontitis group $=209.77 \pm 44.30$, control group $=125.60 \pm 44.16$ ) were significantly higher in the periodontitis group $(\mathrm{P}<0.001)$. Highdensity lipoprotein (HDL) and low-density lipoprotein (LDL) cholesterol levels were higher in the case group, but this difference was not statistically significant. Frequency of pathological values of CHL and TG were significantly higher in cases compared with the controls (P $=0.002$ and $\mathrm{P}=0.015$, respectively).

Conclusions: This study indicates that hyperlipidemia may be associated with periodontal disease in healthy individuals; yet whether periodontitis causes an increase in levels of plasma lipids or whether hyperlipidemia is a risk factor for periodontal infection and cardiovascular disease, it needs further investigations.
\end{abstract}

Keywords:Chronic Periodontitis; Hyperlipidemia; Cholesterol; Triglycerides

\section{Background}

Chronic periodontitis is one of the most common inflammatory diseases that lead to destruction of the periodontal ligaments and loss of the adjacent bone and teeth (1). With the progression of periodontitis, bacteria and their endotoxins (lipopolysaccharide) and other products enter the periodontal tissue and blood circulation causing, systemic or local inflammatory reactions in the host. Also, periodontal disease causes Th1 reaction and releases cytokines such as tumor necrosis factor- $\alpha$ (TNF- $\alpha$ ) and interleukin-1 (IL-1 $\beta)$, which can increase the risk of coronary heart disease (2).

Fentoglu et al. reported that serum and gingival cervical fluid (GCF) levels of TNF- $\alpha$, IL-1 $\beta$ and IL-6 seem to be relating factors between periodontal disease and high serum lipid levels (3). Several studies have shown that there is a relationship between periodontitis and high serum lipid levels (4-9), while other studies have found no such relationship (10-14). Losche et al., Cutler et al., and Moeintaghavi et al. concluded that plasma levels of lipids in individuals with periodontitis were significantly higher than healthy periodontal subjects $(4,7,8)$. Hamissi et al. on the other hand, reported no significant difference be- tween levels of total cholesterol (CHL), triglycerides (TG), high-density lipoprotein (HDL) and low-density lipoprotein (LDL) cholesterol in periodontitis subjects compared with controls (14).

\section{Objectives}

According to the controversy between different studies, this study was performed to determine the relationship between chronic periodontitis and blood serum lipid levels.

\section{Patients and Methods}

This case-control study was conducted at the school of dentistry, Ahvaz Jundishapur University of Medical Sciences, Ahvaz, Iran, during March 2011. The study protocol was approved by the ethics committee (Number: 216/36/ MP/D). From the patients that referred to the periodontics department, 100 subjects with chronic periodontitis that had at least one periodontal pocket with $4 \mathrm{~mm}$ depth in every quadrant and showed bone destruction in their radiography, were selected on the basis of the simple ran- 
dom sampling method, and then based on the exclusion criteria 45 subjects were excluded from the case group. Also, 45 periodontally healthy subjects that did not meet the exclusion criteria were selected for the control group by the simple random sampling method, from 100 individuals referred to the oral medicine department. According to previous studies and the statistical formula, in total 90 subjects ( 45 cases and 45 controls) were selected to participate in the study; the significance of the study was set at $\alpha=0.05$ and the power was set at $80 \%$.

$$
n=\frac{\left(Z_{1-\frac{\alpha}{2}}+Z_{1-\beta}\right)^{2}\left(\sigma_{1}^{2}+\sigma_{2}^{2}\right)}{\left(\text { micro } ;_{1}-\text { micro } ;_{2}\right)^{2}}=
$$

These two groups were matched for confounding factors such as gender, age and number of teeth. All participants signed an informed consent form. One periodontist performed all periodontal examinations in a dental chair using a dental mirror and a world health organization (WHO) periodontal probe according to the WHO instructions. Clinical oral examinations included assessment of the periodontal pockets in all teeth except the third molars. Dental and medical history was determined on the basis of information obtained from the health interviews and examinations. Exclusion criteria were periodontal treatment in the last six months, diseases such as cancer, cardiovascular disease and diabetes mellitus or any endocrine disease, hypertension, use of alcohol, tobacco smoking, drug treatment for hyperlipidemia and excessive obesity (BMI $\geq 30 \mathrm{~kg} / \mathrm{m}^{2}$ ). Body mass index(BMI) was calculated as body weight $(\mathrm{kg})$ divided by height $\left(\mathrm{m}^{2}\right)(15)$.

All subjects were referred to the central laboratory of Golestan Hospital where fasting blood samples were collected to determine serum lipid levels, using routine enzymatic methods (Autoanalyzer, BT 3000, Italy), which were calibrated daily by the operator. The pathological cut-off points according to the laboratory's recommendations were as follows: total cholesterol (CHL) $>220 \mathrm{mg} /$ $\mathrm{dL}$, triglycerides $(\mathrm{TG})>200 \mathrm{mg} / \mathrm{dL}$, low density lipoproteins (LDL) $>178 \mathrm{mg} / \mathrm{dL}$ and high density lipoproteins $(\mathrm{HDL})<29 \mathrm{mg} / \mathrm{dL}$.

\subsection{Statistical Analysis}

The data were tested for normal distribution and were found to be normally distribution (Kolmogorov-Smirnov test). Student t-test and chi-square test were applied for intergroup comparisons between the variables (serum lipid levels), and the significance level was set at $\alpha \leq 0.05$. Also, the odds ratio with $95 \%$ confidence interval was estimated. Statistical analysis was performed using the SPSS software (version 17, Chicago, IL, USA).

\section{Results}

Ninety subjects participated in the study, including 45 cases (20 males, 25 females) of chronic periodontitis and
45 controls ( 22 males, 23 females). The clinical characteristics of the case and control groups are illustrated in Table 1. Mean values and standard deviations of the serum levels of TG, total cholesterol, HDL and LDL cholesterol in both groups are illustrated in Table 1. Mean values of TG in the periodontitis group $(209.77 \pm 44.30)$ were significantly higher compared to the control group (125.60 \pm 44.16$)$, $\mathrm{P}<0.001$. Mean values of CHL were significantly higher in the periodontitis group $(218.11 \pm 29.77)$ compared to the healthy group $(162.31 \pm 48.24), \mathrm{P}<0.001$.

Low-density lipoproteins and HDL cholesterol were higher in the case group, yet this difference was not significant. Body mass index was not significantly higher in periodontal patients than control individuals. The frequency of abnormal serum lipid levels in both groups is presented in Table 2. The frequency of pathological TG and CHL was significantly higher in cases compared to controls ( $\mathrm{P}=0.015, \mathrm{P}=0.002$, respectively). Odds ratio of periodontal disease for hypertriglyceridemia and hypercholesterolemia were estimated as 5.2 and 4.04, respectively.

Table 1. Information on Individuals of the Periodontitis and Control Groups $(\mathrm{n}=45)^{\mathrm{a}, \mathrm{b}}$

\begin{tabular}{|c|c|c|c|}
\hline Variable & $\begin{array}{c}\text { Periodontitis } \\
\text { Group }\end{array}$ & $\begin{array}{l}\text { Control } \\
\text { Group }\end{array}$ & $\begin{array}{l}\text { Students t- } \\
\text { Test PValue }\end{array}$ \\
\hline Age, $y$ & $34.11 \pm 4.71$ & $35.36 \pm 5.05$ & 0.23 \\
\hline $\begin{array}{l}\text { Body mass } \\
\text { index, } \mathrm{kg} / \mathrm{m}^{2}\end{array}$ & $25.17 \pm 2.5$ & $23.73 \pm 3.1$ & 0.46 \\
\hline $\begin{array}{l}\text { Total choles- } \\
\text { terol, mg/dL }\end{array}$ & $218.11 \pm 29.77$ & $162.31 \pm 48.24$ & $<0.001^{\mathrm{C}}$ \\
\hline $\begin{array}{l}\text { Triglycerides, } \\
\text { mg/dL }\end{array}$ & $209.77 \pm 44.30$ & $125.60 \pm 44.16$ & $<0.001^{\mathrm{C}}$ \\
\hline LDL, mg/dL & $116.97 \pm 37.41$ & $115.68 \pm 41.53$ & 0.87 \\
\hline HDL, mg/dL & $55.24 \pm 14.05$ & $53.75 \pm 17.87$ & 0.66 \\
\hline
\end{tabular}

Table 2. Frequency of Pathological Values of Serum Lipids in Controls and Periodontitis Patients $(n=45)^{a, b}$

\begin{tabular}{|c|c|c|c|c|}
\hline $\begin{array}{l}\text { Cut-off, mg/ } \\
\text { dL }\end{array}$ & Value & $\begin{array}{l}\text { Control } \\
\text { Group }\end{array}$ & $\begin{array}{c}\text { Periodontitis } \\
\text { Group }\end{array}$ & $\begin{array}{c}\text { Chi-Square Test } \\
\text { PValue }\end{array}$ \\
\hline CHL & $>220$ & $8(17.7)$ & $21(46.6)$ & $0.002^{c}$ \\
\hline $\begin{array}{l}\text { Triglycer- } \\
\text { ides }\end{array}$ & $>200$ & $6(13.3)$ & $20(44.4)$ & $0.015^{c}$ \\
\hline LDL & $>178$ & $2(4.44)$ & $3(6.66)$ & 0.62 \\
\hline HDL & $<29$ & $2(4.44)$ & $3(6.66)$ & 0.62 \\
\hline
\end{tabular}




\section{Discussion}

This study showed that total cholesterol and TG levels were significantly higher in periodontal patients compared to healthy subjects, yet LDL and HDL cholesterol levels were not significantly different between the two groups. The odds ratio for having high TG and CHL levels in patients with chronic periodontitis was 5.2 and 4.04 times higher than healthy periodontal subjects. Other studies have confirmed a relationship between chronic periodontitis and serum lipid levels (4-9), yet these studies had different study designs, samples sizes, and different methodologies for diagnosing periodontal disease and assaying serum lipid levels. On the other hand, some studies have rejected this relationship (10-14).

Ebersole et al. reported that periodontal disease causes a significant rise in serum TG, CHL, HDL and LDL levels in non-human primates (16). Cutler et al. observed that chronic periodontitis has a significant relationship with the increase of serum TG and total cholesterol, which agrees with our study. Their study estimated an odds ratio of 8.6 and 7 for hypertriglyceridemia and hypercholesterolemia, which was higher than our study (5.2 for TG and 4.04 for CHL) (7).

In a study carried out by Losche et al., pathological levels of TG and total cholesterol was higher in periodontal patients compared to healthy subjects, similar to the findings of this study, yet they also reported that periodontal patients had a significantly higher abnormal LDL levels (4). A possible explanation could be the difference in the definition of abnormal levels of LDL; in the study carried out by Losche et al. LDL $>160 \mathrm{mg} / \mathrm{dL}$ and HDL $<45 \mathrm{mg} / \mathrm{dL}$ was considered pathological (4) yet in our study LDL $>178 \mathrm{mg} / \mathrm{dL}$ and HDL $<29 \mathrm{mg} / \mathrm{dL}$ was considered pathological by the laboratory; therefore less patients were in this range.

Katz et al. confirmed a positive relationship between deep periodontal pockets and high total cholesterol and LDL levels, using the index Community Periodontal Index of Treatment Needs (CPITN) (5, 6). Moeintaghavi et al. and Taleghani et al. reported similar results to this study, yet the odds ratio for high TG and CHL in this study was lower than that reported by Moeintaghavi ( 6.7 for TG and 5.9 for cholesterol) $(8,9)$. Machado et al. concluded that mean levels of cholesterol and TG were higher in patients with periodontitis yet the difference was not significant (10). Saxlin et al. carried out a cross-sectional study on 1297 subjects and concluded that serum lipid levels were not associated with an increased likelihood of periodontal infection among normal weight subjects, yet were associated with the presence of deepened periodontal pockets (4 mm or more) among obese subjects (11). Sridhar et al. rejected the relationship between periodontal disease, coronary heart disease and serum lipid levels (12). Hamissi et al. carried out a case-control study where the case and control groups were matched by, age, gender, diet, weight, height, health condition and number of teeth. They concluded that serum lipid levels in the case group were higher than the control group yet this difference was not significant. They claimed that the difference between their results and other studies was due to samples size and the severity of periodontitis in the case group (14). Noack et al. stated that the relationship between hyperlipidemia and periodontal infection could be due to polymorphonuclear leucocytes (PMN-cells) dysfunction (17).

Periodontitis is an infectious disease caused by gramnegative anaerobic bacteria. It has been shown that acute infections can interrupt lipid metabolism, and there is a significant rise in plasma TG during gram-negative bacterial infections (18). It seems that these changes are due to cytokines such as TNF- $\alpha$, IL- $1 \beta$ and IL- 6 which are produced in large amounts in infectious periodontal tissues (19-21). These cytokines increase the mobilization of lipids from the liver and adipose tissue (22), and increase the binding of LDL to endothelium and smooth muscles (23). It has been reported that oxidative modification of LDL leads to an increase in cholesterol accumulation because modified LDL is very susceptible to macrophage uptake (24). On the other hand, several studies have reported the possible role of hyperlipidemia in periodontitis progression (25). It has been shown that hyperlipidemia causes an increase in white blood cell (WBC) activity $(26,27)$, which helps the progression of periodontal disease in adults $(28,29)$. This study was carried out on non-smoking subjects thus confounding relationships with the toxic effects of smoking were omitted. We also excluded the diabetic subjects to omit the confounding effects of diabetes. Subjects with BMI $\geq 30$ were also excluded because many studies report a relationship between obesity and periodontal infections (30-32). The case and control groups were matched by age, because older age is a risk factor for hyperlipidemia. This study showed a relationship between periodontitis and hyperlipidemia yet whether alterations in lipid metabolism are the cause or result of periodontitis cannot be determined. Therefore we suggest that cohort studies with more samples should be carried out to better understand this relationship.

Hyperlipidemia can lead to cardiovascular diseases, which have high mortality rates. This study, similar to some other previous reports, proves a relationship between periodontal disease and hyperlipidemia. Therefore, it is recommended that patients with heart disease should strongly consider periodontal treatment.

\section{Acknowledgements}

We thank the Ahvaz Jundishapur University of Medical Sciences for providing the financial support for this study. We also specially thank Dr. Alireza Haerian Ardekani for assisting with the editing of this paper.

\section{Authors' Contributions}

Supervisor: Leila Golpasand Hagh; advisor: Faramarz Zakavi; data collection and analysis: Fatemeh Hajizadeh and Morteza Saleki ; drafting of the manuscript: Fatemeh Hajizadeh and Morteza Saleki. 


\section{Funding/Support}

This study was supported by a teaching and research scholarship from the School of Dentistry, Ahvaz Jundishapur University of Medical Sciences, Ahvaz, Iran. The study was approved by the Medical Ethics and Research Office at the Ahvaz Jundishapur University of Medical Sciences and financially supported by this University.

\section{References}

1. 1999 International International Workshop for a Classification of Periodontal Diseases and Conditions. Papers. Oak Brook, Illinois, October 30-November 2,1999. Ann Periodontol. 1999;4(1):i.

2. Kinane DF. Periodontal diseases' contributions to cardiovascular disease: an overview of potential mechanisms. Ann Periodontol. 1998;3(1):142-50.

3. Fentoglu O, Kirzioglu FY, Ozdem M, Kocak H, Sutcu R, Sert T. Proinflammatory cytokine levels in hyperlipidemic patients with periodontitis after periodontal treatment. Oral Dis. 2012; 18(3):299-306.

4. Losche W, Karapetow F, Pohl A, Pohl C, Kocher T. Plasma lipid and blood glucose levels in patients with destructive periodontal disease. J Clin Periodontol. 2000;27(8):537-41.

5. Katz J, Chaushu G, Sharabi Y. On the association between hypercholesterolemia, cardiovascular disease and severe periodontal disease. J Clin Periodontol. 2001;28(9):865-8.

6. Katz J, Flugelman MY, Goldberg A, Heft M. Association between periodontal pockets and elevated cholesterol and low density lipoprotein cholesterol levels. J Periodontol. 2002;73(5):494-500.

7. Cutler CW, Shinedling EA, Nunn M, Jotwani R, Kim BO, Nares S, et al. Association between periodontitis and hyperlipidemia: cause or effect? J Periodontol.1999;70(12):1429-34.

8. Moeintaghavi A, Haerian-Ardakani A, Talebi-Ardakani M, Tabatabaie I. Hyperlipidemia in patients with periodontitis. J Contemp Dent Pract. 2005;6(3):78-85.

9. Taleghani F, Shamaei M, Shamaei M. Association between chronic periodontitis and serum lipid levels. Acta Med Iran. 2010; 48(1):47-50.

10. Machado AC, Quirino MR, Nascimento LF. Relation between chronic periodontal disease and plasmatic levels of triglycerides, total cholesterol and fractions. Braz Oral Res. 2005;19(4):284-9.

11. Saxlin T, Suominen-Taipale L, Kattainen A, Marniemi J, Knuuttila M, Ylostalo P. Association between serum lipid levels and periodontal infection. J Clin Periodontol. 2008;35(12):1040-7.

12. Sridhar R, Byakod G, Pudakalkatti P, Patil R. A study to evaluate the relationship between periodontitis, cardiovascular disease and serum lipid levels. Int J Dent Hyg. 2009;7(2):144-50

13. Korhonen S, Saxlin T, Suominen L, Jula A, Knuuttila M, Ylostalo P. Serum cholesterol ratios and periodontal infection: results of the Health 2000 Survey. J Clin Periodontol. 2011;38(9):787-94.

14. Hamissi J, Shahsavarani MT, Hamissi H. A Comparison of Serum
Lipid Profile between Periodontitis Patients and Healthy Individuals. Iran Red Crescent Med J. 2011;13(4):283-4.

15. Eknoyan G. Adolphe Quetelet (1796-1874)-the average man and indices of obesity. Nephrol Dial Transplant. 2008;23(1):47-51.

16. Ebersole JL, Cappelli D, Mott G, Kesavalu L, Holt SC, Singer RE. Systemic manifestations of periodontitis in the non-human primate. J Periodontal Res. 1999;34(7):358-62.

17. Noack B, Jachmann I, Roscher S, Sieber L, Kopprasch S, Luck C, et al. Metabolic diseases and their possible link to risk indicators of periodontitis. J Periodontol. 2000;71(6):898-903.

18. Wanner C, Zimmermann J, Quaschning T, Galle J. Inflammation, dyslipidemia and vascular risk factors in hemodialysis patients. Kidney Int Suppl. 1997;62:S53-5.

19. Beck JD, Offenbacher S, Williams R, Gibbs P, Garcia R. Periodontitis: a risk factor for coronary heart disease? Ann Periodontol. 1998;3(1):127-41.

20. Valtonen VV. Infection as a risk factor for infarction and atherosclerosis. Ann Med.1991;23(5):539-43.

21. Feingold KR, Grunfeld C. Tumor necrosis factor-alpha stimulates hepatic lipogenesis in the rat in vivo.J Clin Invest.1987;80(1):184-90.

22. Iacopino AM, Cutler CW. Pathophysiological relationships between periodontitis and systemic disease: recent concepts involving serum lipids. J Periodontol. 2000;71(8):1375-84.

23. Hajjar DP, Haberland ME. Lipoprotein trafficking in vascular cells. Molecular Trojan horses and cellular saboteurs. J Biol Chem. 1997;272(37):22975-8.

24. Piotrowski JJ, Hunter GC, Eskelson CD, Dubick MA, Bernhard VM. Evidence for lipid peroxidation in atherosclerosis. Life Sci. 1990;46(10):715-21.

25. Scardina GA, Pisano T, Cacioppo A, Messina P. Periodontal alteration of the microcirculation and hypercholesterolemia: a possible correlation? South Med J. 2011;104(2):116-20.

26. Croft KD, Beilin LJ, Vandongen R, Rouse I, Masarei J. Leukocyte and platelet function and eicosanoid production in subjects with hypercholesterolaemia. Atherosclerosis. 1990;83(2-3):101-9.

27. Krause S, Pohl A, Pohl C, Liebrenz A, Ruhling K, Losche W. Increased generation of reactive oxygen species in mononuclear blood cells from hypercholesterolemic patients. Thromb Res. 1993;71(3):237-40.

28. Krause S, Brachmann P, Brandes C, Losche W, Hoffmann T, Gangler P. Aggregation behaviour of blood granulocytes in patients with periodontal disease. Arch Oral Biol. 1990;35(1):75-7.

29. Gustafsson A, Asman B. Increased release of free oxygen radicals from peripheral neutrophils in adult periodontitis after Fc deltareceptor stimulation. J Clin Periodontol.1996;23(1):38-44.

30. Saito T, Shimazaki Y, Koga T, Tsuzuki M, Ohshima A. Relationship between upper body obesity and periodontitis. J Dent Res. 2001;80(7):1631-6.

31. Genco RJ, Grossi SG, Ho A, Nishimura F, Murayama Y. A proposed model linking inflammation to obesity, diabetes, and periodontal infections. J Periodontol. 2005;76(11 Suppl):2075-84.

32. Sarlati F, Akhondi N, Ettehad T, Neyestani T, Kamali Z. Relationship between obesity and periodontal status in a sample of young Iranian adults. Int Dent J. 2008;58(1):36-40. 